\section{OC15 PAEDIATRIC MILD TRAUMATIC BRAIN INJURY IS ASSOCIATED WITH SYSTEMIC INFLAMMASOME ACTIVATION AND PUBERTAL SCORING}

\footnotetext{
1,2Emer Ryan*, 'Lynne Kelly, ${ }^{1}$ Eimear Duff, ${ }^{2}$ Turlough Bolger, ${ }^{1,2,3,4}$ Eleanor Molloy. ${ }^{1}$ Discipline of Paediatrics, Department of Medicine, Trinity College, Dublin, Ireland; ${ }^{2}$ Tallaght University Hospital, Dublin, Ireland; ${ }^{3}$ The Coombe Women's Hospital, Dublin, Ireland; ${ }^{4}$ National Children's Research Centre, Crumlin, Dublin, Ireland
}

\subsection{6/archdischild-2019-epa.15}

Background Mild Traumatic Brain Injury (mTBI) is a common childhood occurrence with a more severe phenotype in adolescence. Systemic inflammation has been demonstrated to be integral to the pathogenesis of ongoing symptoms. The inflammasome is a component of innate immunity that is involved in regulating and inducing inflammation in response to cell damage and may have a modifiable role in mTBI.

Objective We demonstrated inflammasome pathway activation in response to $\mathrm{mTBI}$. We hypothesized that pubertal development modulates inflammasome activation. We correlated pubertal scores with inflammasome gene transcription.

Methods Children were recruited to the study following presentation to the emergency department with symptomatic head injuries (GCS 14/15; $\mathrm{n}=21$ ) and compared to age-matched Controls $(\mathrm{n}=10)$. Pubertal developmental self-rating scales (PDS) with 5 rating questions were administered with a maximum score of 20. mRNA was extracted from whole blood and the Inflammasome components [Interleukin (IL)- 1beta and NLR Family Pyrin Domain Containing 3 (NLRP3)] in mTBI and controls were profiled with RT-PCR analysis on the ABI 7900.

Results The mean age of children recruited was $12.0( \pm 3.9)$ years with 18 males. Both NLRP3 and IL-1 $\beta$ were significantly raised in the mTBI group versus controls $(p<0.0001)$. IL-1 $\beta$ was significantly downregulated at 2 weeks post TBI $(p<0.0001)$. There was a significant correlation between pubertal score and IL-1 $\beta$ in mTBI $(p=0.04)$.

Conclusion Inflammasome activation via the NLRP3 pathway is important in paediatric mTBI. IL1beta activation was more prominent in children at more advanced stages of puberty. The interplay of pubertal maturity and the innate immune system may explain the greater burden of symptoms in patients during adolescence.

\section{OC16 FEVER AS RISK FACTOR OF COMPLICATION IN CHILDREN HOSPITALIZED DUE TO RSV INFECTION}

1,2Małgorzata Czajkowska*, 1,2August Wrotek, 1,2Teresa Jackowska. 'Department of Pediatrics, the Medical Centre of Postgraduate Education, Marymoncka 99/103, 01-813, Warsaw, Poland; '2Department of Pediatrics, Bielanski Hospital, Cegłowska 80,01-809, Warsaw, Poland

\subsection{6/archdischild-2019-epa.16}

Background Frequency of complications in children hospitalized due to Respiratory Syncytial Virus (RSV) infectionsis high,and fever is supposed to correlatewith the risk of complications. The aim of the study was to assess the correlation between presence of fever, its magnitude, and durationand the risk of complications.

Material and methods 221 children (10 days to 91 months; median 3 months) were hospitalized due to RSV infection (2016/2017 and 2017/2018 seasons).219 children met inclusion criteria (1patient discharged on parents' request, and 1 with nosocomial infection were excluded). RSV infections were diagnosed with rapid antigen test and/or polymerase chain reaction. Acute otitis media(AOM) was diagnosed with otoscopy; pneumonia was confirmed with chest X-ray.We analyzed the highest fever, duration of fever, time of its presence (prior or during hospitalization).

Results Fever was present in 40\%(88/219) patients, including 69 cases of fever prior and 55 during hospitalization (36 cases prior and during hospitalization). The risk of complications was higher in patients with fever $(\mathrm{OR}=6.09$, 95\%CI: $3.3-$ 11.2), and it was more clearly pronounced in patients with longer (at least 3 days) duration of fever $(\mathrm{OR}=9.04,95 \% \mathrm{CI}$ : 4.2-19.4) than in patients with higher (at least $39^{\circ} \mathrm{C}$ ) fever $(\mathrm{OR}=2.2$, 95\%CI: 0.7-6.5). Presence of fever was related with higher risk of both pneumonia andAOM $(\mathrm{OR}=5.27$, 95\%CI: 2.7-10.4, and $\mathrm{OR}=3.43$, 95\%1.5-7.7 respectively). Longer fever duration correlated with pneumonia more significantly than higher fever $(\mathrm{OR}=6.34,95 \% \mathrm{CI}: 3.1-13.1$ vs. $\mathrm{OR}=0.94,95 \% \mathrm{CI}: 0.4-2.5)$. Similar correlation was observed in $\mathrm{AOM}(4.53,95 \% \mathrm{CI}$ : 2-10.2 for longer vs. $\mathrm{OR}=1.96,95 \%$ CI: $0.7-5.6$ for higher fever). Presence of fever showed AUC $=0.76$ (95\%CI: $0.69-0.83 ; \mathbf{p}<0.001)$ with cut-off value of 3 days showing $44 \%$ sensitivity and 91\%specificity; $78 \%$ and $70 \%$ positive and negative predictive value respectively.

Conclusions Fever correlates with the risk of complications, but duration of fever seems to be crucial in terms of pneumonia and AOM.

\section{OC17 EVALUATION OF CIRCADIAN MELATONIN SECRETION IN OBESE ADOLESCENTS}

Olga Berdina, Irina Madaeva, Leonid Sholokhov, Svetlana Bolshakova, Maria Rashidova, Lyubov Rychkova*. Scientific Centre for Family Health and Human Reproduction Problems, Irkutsk, Russian Federation

\subsection{6/archdischild-2019-epa.17}

Background and aims Obesity has been described as the epidemic of the 21st century. It is known that obesity may lead to a change in the main circadian rhythm - sleep-wake cycle, in which the melatonin (MLT) plays an especially important role, but little is known about circadian melatonin secretion in obese adolescents. The aim of this study was to measure 4point saliva melatonin levels in obese adolescents and investigate circadian melatonin secretion relationship with obesity.

Materials and methods We studied 30 obese adolescents and 10 normal weight (NW) adolescents aged 15-17 year. Obesity and NW were determined if body mass index (BMI) Zscore $>2$ and BMI Z-score $<1$ for age and sex, respectively. All adolescents underwent polysomnography with the use of GRASS-TELEFACTOR Twin PSG (Comet) with As40 amplifier and an integrated module for sleep SPM-1 (USA) in order to verify any present sleep disorders. 18 obese adolescents with sleep apnea were excluded from the study. Non-stimulated saliva was used to determine the melatonin levels in 6.00-7.00, $12.00-13.00,18.00-19.00$ and 23.00-00.00 hours by an immunoenzymometric assay using Buhlmann commercial sets (Switzerland) on the EL $\times 808$ Micro Plate Reader (USA). All differences were considered significant at $p<0.05$.

Results There were no significant differences related to age and sex between obesity and NW groups. A comparative analysis of the melatonin level in adolescents of main and control groups showed significantly lower melatonin levels both in evening hours $(0.54 \pm 0.51 \mathrm{pg} / \mathrm{ml}$ vs $2.13 \pm 1,88 \mathrm{pg} / \mathrm{ml}$, 